\title{
Raça e Saúde Mental no Ensino Superior: Uma revisão sistemática
}

\author{
Race and Mental Health in Higher Education: A systematic review \\ Raza y Salud Mental en la Educación Superior: Una revisión sistemática
}

\section{Resumo}

Objetivo: Apresentar resultados de Revisão Sistemática da literatura científica que trata como estigmas de raça/cor da pele influenciam na saúde mental dos estudantes universitários negros. Metodologia: A pesquisa foi realizada nas bases de dados SciELO, Lilacs e PubMed; os descritores usados foram: "saúde mental", "estudantes" e "raça", nas bases SciELO e Lilacs, e "mental health", "students" e "race" na base PubMed. Os filtros utilizados foram: artigos em português e inglês, texto completo, publicados no ano de 2015 até 2020. Resultados: Realizada a busca, um estudo brasileiro e 12 norte-americanos respondiam à questão norteadora proposta, tendo sido selecionados para análise. A maioria dos estudos (08) eram transversais quantitativos, utilizando questionários na investigação proposta. Além desses, identificou-se uma meta-síntese interpretativa qualitativa; um estudo transversal baseado em entrevista semiestruturada; e três estudos longitudinais. Conclusão: Torna-se evidente diante de tal pesquisa a raridade de artigos que incluam a variável raça nas análises sobre saúde mental de estudantes universitários; isso denota negligência às especificidades enfrentadas pelo estudante negro em função do racismo. A escassez percebida motiva novos estudos sobre a dimensão do problema e sobre possíveis soluções para inclusão e formação de melhores profissionais.

Palavras-chave: População negra; Saúde mental; Estudantes; Ensino.

\begin{abstract}
Objective: To present the results of a systematic review of the scientific literature that deals with how race/skin color stigmas influence the mental health of black university students. Methodology: The search was carried out in SciELO, Lilacs and PubMed databases; the descriptors used were: "mental health", "students" and "race", in the SciELO and Lilacs databases, and "mental health", "students" and "race" in the PubMed database. The filters used were: articles in Portuguese and English, full text, published in the year 2015 until 2020. Results: After the search, a Brazilian study and 12 North Americans answered the proposed guiding question and were selected for analysis. Most studies (08) were quantitative cross-sectional, using questionnaires in the proposed investigation. In addition to these, a qualitative interpretive meta-synthesis was identified; a cross-sectional study based on semi-structured interviews; and three longitudinal studies. Conclusion: The rarity of articles that include the variable race in the analyzes of mental health
\end{abstract}


among university students becomes evident in this research; this denotes neglect of the specificities faced by the black student due to racism. The perceived scarcity motivates further studies on the dimension of the problem and on possible solutions for inclusion and training of better professionals.

Keywords: Black people; Mental health; Students; Teaching.

\section{Resumen}

Objetivos: Presentar los resultados de la revisión sistemática de la literatura científica que trata sobre cómo estigmas de raza/color de piel influyen en la salud mental de los estudiantes universitarios negros. Metodología: La búsqueda se realizó en las bases de datos SciELO, Lilacs y PubMed; los descriptores utilizados fueron: "saúde mental", "estudantes" y "raça" (portugués) en las bases de datos SciELO y Lilacs, y "mental health", "students" y "race" (inglés) en la base de datos PubMed. Los filtros utilizados fueron: artículos en portugués e inglés, publicados desde el año 2015 hasta el año 2020. Resultados: Al final de la búsqueda, 1 estudio nacional y 12 estudios internacionales, siendo todos de Estados Unidos, respondieron a la pregunta orientadora propuesta y fueron analizados. La mayoría de los estudios (08) fueron cuantitativos y transversales que utilizaron cuestionarios. Además de estos, hay 1 metasíntesis interpretativa cualitativa; 1 estudio transversal que utilizó una entrevista semiestructurada; y 3 estudios longitudinales. Se utilizó 1 estudio nacional (8\%) y 12 estudios internacionales (92\%), siendo todos de Estados Unidos. Conclusión: Se evidencia en esta investigación que es raro que los artículos incluyan la variable raza en los análisis de salud mental de estudiantes universitarios; esto denota que hay un descuido de las especificidades que enfrenta el estudiante negro debido al racismo. La escasez percibida motiva nuevos estudios sobre la dimensión del problema y sobre posibles soluciones para la inclusión y formación de mejores profesionales.

Palabras clave: Población negra; Salud mental; Estudiantes; Enseñanza.

\section{Introdução}

No primeiro trimestre de 2020, cerca de 9,2\% da população brasileira se autodeclarou preta e outros 47,2\% de cor parda, compondo em soma o que o IBGE caracteriza como população negra, conformando, assim, a maioria da população brasileira (IBGE, 2020). No entanto, quando é analisado o perfil dos $10 \%$ mais ricos da população brasileira, em 2018, apenas 27,7\% eram negros. Essa discrepância financeira é evidente mesmo na população em geral, dado que, nesse mesmo ano, o rendimento médio domiciliar per capita das populações branca e negra foi, respectivamente, $\mathrm{R} \$ 1.846$ e $\mathrm{R} \$ 934$ - sendo o primeiro valor aproximadamente o dobro do segundo (IBGE, 2019).

Essa situação é consequência do longo processo de exploração e desumanização da população negra no país, a qual, após séculos de escravidão, deixou - legalmente - de ser mercadoria, embora tenha permanecido marginalizada (Madeira \& Gomes, 2018; Braga \& Milani, 2019). Pois que, como reflexo, inclusive na atualidade, a população negra brasileira continua vítima de discriminação por raça (termo comumente utilizado como sinônimo de cor de pele), restando submetida a relações de poder que mantém indivíduos e coletivos em constante desvantagem, num sistema presente em praticamente toda a estrutura da sociedade brasileira (Madeira \& Gomes, 2018; Batista, 2018).

Com isso, não é estranho verificar que a população negra tenha acesso extremamente limitado à atenção em saúde, agravado por causas diversas como: deficiência na oferta dos serviços públicos ou privados nas comunidades em que vivem e desinformação amparada pelo binômio "baixa escolaridade/baixa cidadania". Além disso, há que se considerar a discriminação sofrida pelas pessoas negras a partir de profissionais de saúde, que impacta negativamente na prestação adequada de serviços e na adesão aos tratamentos propostos (Silva et al, 2020; Oliveira \& Kubiak, 2019). Tais iniquidades motivaram a mobilização de movimentos sociais em prol de políticas públicas que priorizem o combate à desigualdade étnico-racial e ao racismo institucional/estrutural, que colocam a população negra em piores condições de saúde. Esse movimento culminou no reconhecimento desse cenário e na criação da Política Nacional de Saúde Integral da População Negra (PNSIPN), que visa a promoção da saúde integral para esse público no âmbito do SUS (Brasil, 2016).

Como componente indissociável do conceito atualizado de saúde, não há razões para crer que não persistam desigualdades semelhantes em relação aos serviços específicos de atenção à saúde mental. Contudo, a carência de análises relacionando os quesitos raça/cor com dados de saúde mental tornou-se um fator limitante para conclusões apuradas. No 
entanto, as desigualdades socioeconômicas, a violência — a qual a juventude negra é especialmente vulnerável — e toda a discriminação sofrida no dia a dia de pretos e pardos brasileiros são fatores que, certamente, podem desencadear sofrimento psíquico nessas pessoas, em várias intensidades (Brasil, 2016).

Conforme sugerido na revisão sistemática apresentada por Smolen e Araújo (2017), há maior prevalência de transtornos mentais na população negra do que em outros grupos populacionais. Porém, não há unanimidade nesse sentido, e ainda resta a necessidade de serem realizados mais estudos científicos acerca do tema, especialmente em regiões de população negra expressiva, visto que grande parte da literatura sobre transtornos mentais que incluem raça/cor como variável de análise foram realizados regiões Sul e Sudeste, assim, apresentando pouca expressividade de população não-branca nas amostras.

No que se refere aos estudantes universitários negros, pouquíssimos estudos analisam as vulnerabilidades a que eles podem estar mais sujeitos em termos de saúde mental. Nesse contexto, é de grande relevância o estudo de Jochman et al. (2019), o qual analisou as consequências da discriminação na saúde mental de estudantes de uma universidade predominantemente branca dos Estados Unidos. Tal estudo apresenta resultados que corroboram com a literatura pré-existente ao concluir que a discriminação racial gera situações de distresse já em curto prazo, podendo acumular, prejudicando a saúde mental dos estudantes de minorias étnicas por um longo período e aumentando a probabilidade de que suas relações sociais resultem em experiências menos gratificantes e mais frustrantes, inclusive violentas.

Evidencia-se, portanto, a necessidade de estudos acerca da saúde mental de estudantes universitários, ou mesmo da população em geral, que demonstrem a relação dela com a variável raça/cor, especialmente no Brasil, e, de forma ainda mais acentuada, nas regiões de população negra mais expressiva. Dadas as vulnerabilidades a que a população negra está sujeita e as particularidades do cenário nacional, o conhecimento acerca dos potenciais danos à saúde mental aos quais os estudantes universitários negros estão sujeitos é fundamental. Nessa perspectiva, foi a partir de tal necessidade que o objetivo deste estudo foi formulado, consistindo em apresentar os resultados de uma revisão sistemática da literatura científica que trata sobre como aspectos relativos à raça/cor da pele influenciam na saúde mental dos estudantes universitários negros.

\section{Metodologia}

A revisão seguiu a proposta PRISMA (Preferred Reporting Items for Systematic Reviews and Meta-Analyses) e os critérios de elegibilidade PICOS (participantes, intervenção, comparação, resultados e desenho de estudo), nas versões mais atuais propostas, datadas de 2.019 (Donato \& Donato, 2019).

A partir da pergunta norteadora: "Quais são os efeitos do racismo na saúde mental de estudantes negros do nível superior?", os critérios foram: para participantes, estudantes negros, da Educação Superior e independentemente do gênero. Com base em transtornos mentais como intervenção, foi comparada a incidência ou não destes e, para os resultados, verificouse se houve ou não associação com o racismo. Em caso de haver, se foi direta ou inversa. Na associação direta, o racismo perpetuado no Ensino Superior aumentou a incidência de transtornos mentais em estudantes negros; na associação inversa, reduziu ou não alterou a incidência.

Foram selecionados os descritores em português "saúde mental", "estudantes" e "raça" e em inglês "mental health", "students" e "race" a partir da lista de Descritores em Ciências da Saúde (DeCs) e Medical Subject Headings (MeSH) como estratégia para a busca de artigos. A combinação entre operadores booleanos (mental health AND estudantes AND raça) e em inglês (saúde mental AND students AND race) foram aplicada nas bases de dados Lilacs (Literatura Latino-Americana e do Caribe em Ciências da Saúde), PubMed (US National Library of Medicine National Institutes of Health) e SciELO (Scientific Electronic Library Online) com data de busca entre o primeiro dia de 2015 e 31 de dezembro de 2020; almejando-se uma obra final bastante atualizada. A pesquisa nas bases de dados ocorreu em março de 2021. Para o Lilacs e o SciELO, os descritores 
foram pesquisados em português, já para o PubMed foi aplicado o inglês. Da mesma forma, os artigos selecionados estão na língua portuguesa e língua inglesa.

A fim de obter maior segurança na seleção, utilizou-se a categorização da Agency for Healthcare Research and Quality (AHRQ) de 2016. Assim, na busca, filtrou-se artigos até o nível 4 de força de evidência, ou seja, incluindo metanálises de múltiplos estudos controlados, projetos individuais com desenho experi-mental, estudos de coorte, caso-controle e quase experimentais e estudos com desenho não experimental. O nível 5 - relatos de caso - foi excluído pela menor força de evidência. Além disso, submeteu-se a pesquisa e esta foi realizada conforme os requisitos da plataforma PROSPERO (International prospective register of systematic reviews).

Por fim, os artigos foram selecionados e analisados independentemente por 2 autores, a fim de garantir maior segurança durante o processo de busca.

\section{Resultados}

Primeiramente, empregou-se os descritores "saúde mental" e "estudantes" em duas bases de dados distintas — Scielo e o Lilacs - obtendo-se 317 e 231 resultados, respectivamente. Posteriormente, no Scielo, levando em consideração que a proposta inicial do trabalho era estudar o tema no Brasil, empregou-se os filtros: estudos no Brasil; idioma português e data de publicação de 2017 a 2020. Os resultados foram 207, 161 e 70 estudos encontrados em cada uma das etapas, a cada filtro adicionado. Dentre os 70 trabalhos, 19 foram selecionados por título, todavia, nenhum se encaixou à pesquisa proposta após análise dos resumos. Subsequentemente, utilizando-se da base Lilacs com o filtro de Categoria DeCS, não houve resultados encontrados.

Tendo em vista que a busca nas duas primeiras bases de dados não teve sucesso devido à escassez de artigos que respondessem à pergunta proposta nesta pesquisa, os pesquisadores concordaram que uma busca apenas no PubMed seria rasa e optou-se por trocar os descritores, aumentar o período de data de publicação e expandir o estudo para além do Brasil. Tornase evidente diante de tal pesquisa que, embora à primeira vista haja inúmeros artigos sobre a saúde mental de estudantes dentro dos filtros estabelecidos, é raro incluírem a variável raça e, dessa maneira, nota-se negligência às especificidades enfrentadas pelo estudante negro devido ao racismo, evidenciando o abandono deste grupo racial.

Posteriormente à alteração dos descritores para "saúde mental", "estudantes" e "raça", na base de dados SciELO, a busca resultou em 2 artigos. Entretanto, devido ao filtro "ano de publicação" - condizente aos anos de 2015 a 2020 na literatura, como pré-estabelecido na metodologia - a quantidade limitou-se a somente 1 , o qual foi utilizado, conforme apresentado na Figura 1.

Em seguida, no Lilacs, foram encontradas 5 referências no campo "palavras", embora o único resultado encontrado dentro da data pré-estabelecida não se tratasse de alunos universitários, mas escolares. 
Figura 1. Fluxograma da pesquisa.

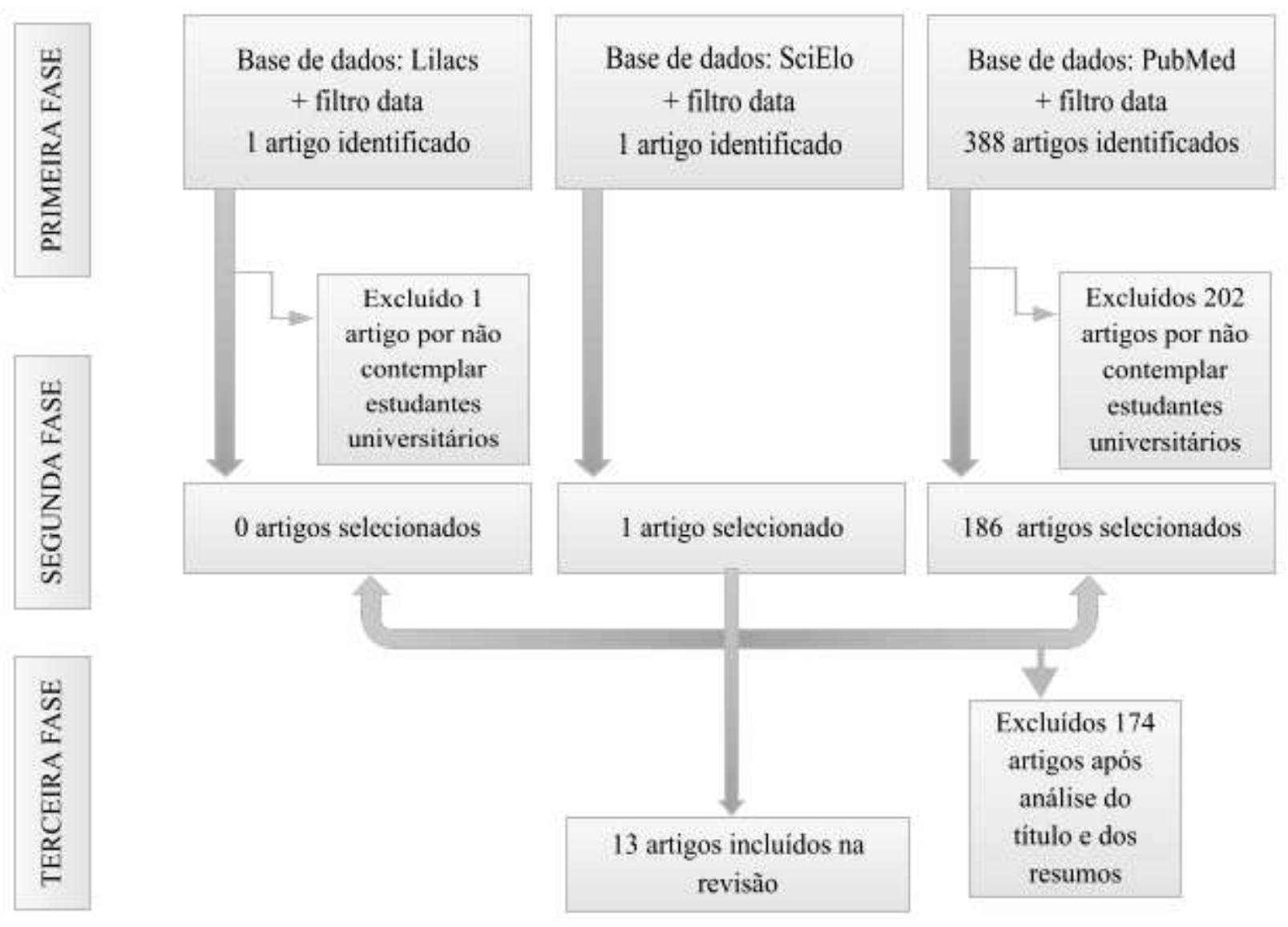

Fonte: Protocolo de pesquisa.

No PubMed, por fim, assim como nota-se na Figura 1, inicialmente, 744 artigos foram obtidos. Contudo, somente 388 após triagem por data. Tendo em conta que a seguinte pesquisa foca em estudantes universitários, a literatura foi reduzida à quantidade de 186. Enfim, 46 estudos pareceram atender aos critérios de elegibilidade do PICOS pré-estabelecidos a partir de seu título, no entanto, apenas 12 contemplaram os objetivos dos autores após análise do resumo. Dessa forma, no resultado final da busca, 13 artigos respondiam à questão norteadora proposta e foram analisados para a discussão.

Outrossim, os 13 artigos em pauta têm algumas de suas características expostas nas Tabelas a seguir. Na Tabela 1 estão expostos ano, título, país de origem do trabalho e autoria. Na planilha 2 repetiu-se o título, como referência, acompanhando a população alvo, resultados, intervenções e variáveis, seguida de resultados. Optou-se por organizar dessa maneira os achados em função do espaço de visualização. 
Tabela 1. Artigos selecionados para inclusão na revisão sistemática: ano, país e autores.

\begin{tabular}{|c|c|c|c|}
\hline Ano & Título & País & Autores \\
\hline 2015 & $\begin{array}{l}\text { Do Dimensions of Ethnic Identity Mediate the } \\
\text { Association Between Perceived Ethnic Group } \\
\text { Discrimination and Depressive Symptoms? }\end{array}$ & Estados Unidos da América & Aerika et al. \\
\hline 2015 & $\begin{array}{l}\text { A delay-discounting measure of preference for } \\
\text { racial/ethnic matching in psychotherapy. }\end{array}$ & Estados Unidos da América & Swift et al. \\
\hline 2016 & $\begin{array}{l}\text { Risk factors and outcomes of chronic sexual } \\
\text { harassment during the transition to college: } \\
\text { Examination of a two-part growth mixture model. }\end{array}$ & Estados Unidos da América & McGinley et al. \\
\hline 2017 & $\begin{array}{l}\text { Addressing Mental Health Needs: Perspectives of } \\
\text { African Americans Living in the Rural South. }\end{array}$ & Estados Unidos da América & Haynes et al. \\
\hline 2017 & $\begin{array}{l}\text { Alcohol Use and Mental Health Conditions } \\
\text { Among Black College Males: Do Those } \\
\text { Attending Postsecondary Minority Institutions } \\
\text { Fare Better Than Those at Primarily White } \\
\text { Institutions? }\end{array}$ & Estados Unidos da América & Barry et al. \\
\hline 2018 & $\begin{array}{l}\text { Racial/ethnic variation in mental health correlates } \\
\text { of substance use among college students. }\end{array}$ & Estados Unidos da América & Sumstine et al. \\
\hline 2019 & $\begin{array}{l}\text { Physical and Mental Health Experiences among } \\
\text { African American College Students. }\end{array}$ & Estados Unidos da América & Barnett et al. \\
\hline 2019 & $\begin{array}{l}\text { Bicultural Identity Harmony and American } \\
\text { Identity Are Associated With Positive Mental } \\
\text { Health in U.S. Racial and Ethnic Minority } \\
\text { Immigrants. Cultural Diversity and Ethnic } \\
\text { Minority Psychology. }\end{array}$ & Estados Unidos da América & Tikhonov et al. \\
\hline 2019 & $\begin{array}{l}\text { Psychiatric Symptoms and Diagnoses Among } \\
\text { U.S. College Students: A Comparison by Race } \\
\text { and Ethnicity. }\end{array}$ & Estados Unidos da América & Chen et al. \\
\hline 2019 & $\begin{array}{l}\text { Mental Health Outcomes of Discrimination } \\
\text { among College Students on a Predominately } \\
\text { White Campus: A Prospective Study. }\end{array}$ & Estados Unidos da América & Jochman et al. \\
\hline 2019 & $\begin{array}{l}\text { Análise da Qualidade e Estilo de Vida entre } \\
\text { Acadêmicos de Medicina de uma Instituição do } \\
\text { Norte do Paraná. }\end{array}$ & Brasil & Buhrer et al. \\
\hline 2019 & $\begin{array}{l}\text { Differences in Hope, Core Self-Evaluations, } \\
\text { Emotional Well-Being, and Health Risk } \\
\text { Behaviors in Freshman University Students. }\end{array}$ & Estados Unidos da América & Griggs e Crawford. \\
\hline 2020 & $\begin{array}{l}\text { Promising Results from the Use of a Korean } \\
\text { Drama to Address Knowledge, Attitudes, and } \\
\text { Behaviors on School Bullying and Mental Health } \\
\text { among Asian American College-Aged Students. }\end{array}$ & Estados Unidos da América & Park et al. \\
\hline
\end{tabular}

Fonte: Autores.

$\mathrm{Na}$ Tabela 1, verifica-se que foram utilizados 1 estudo nacional (8\%) e 12 internacionais (92\%), sendo todos dos Estados Unidos. Desses estudos, apenas um estava na língua portuguesa e, os demais, na língua inglesa. 
Tabela 2. Artigos selecionados para inclusão na revisão sistemática: população estudada, intervenção e variáveis e resultados.

\begin{tabular}{|c|c|c|c|}
\hline Título & População estudada & Intervenção e variáveis & Resultados \\
\hline $\begin{array}{l}\text { Physical and Mental } \\
\text { Health Experiences } \\
\text { among African American } \\
\text { College Students. }\end{array}$ & $\begin{array}{l}\text { tes universitários } \\
\text { ericanos. }\end{array}$ & $\begin{array}{l}\text { Os autores são os principais } \\
\text { instrumentos. Os bancos de dados } \\
\text { Google Scholar e Academic Search } \\
\text { foram revistados para estudos sobre } \\
\text { universitários afro-americanos, grupos } \\
\text { de foco, entrevistas, etnografia, teoria } \\
\text { fundamentada, fenomenologia, dieta, } \\
\text { obesidade, peso e exercícios. }\end{array}$ & $\begin{array}{l}\text { Os resultados revelaram dois temas } \\
\text { abrangentes: (1) percepções da saúde física e } \\
\text { (2) percepções da saúde mental. Cada tema } \\
\text { incluiu três dimensões. As dimensões das } \\
\text { percepções de saúde física incluem (1) } \\
\text { exercícios para benefícios à saúde, (2) } \\
\text { exercícios para a estética e (3) nutrição versus } \\
\text { alimentação saudável. As dimensões das } \\
\text { percepções da saúde mental incluem (1) } \\
\text { estresse, (2) confiança e (3) espiritualidade. }\end{array}$ \\
\hline $\begin{array}{lr}\text { Alcohol Use and Mental } \\
\text { Health } \text { Conditions } \\
\text { Among Black College } \\
\text { Males: Do Those } \\
\text { Attending Postsecondary } \\
\text { Minority Institutions } \\
\text { Fare Better Than Those } \\
\text { at Primarily White } \\
\text { Institutions? }\end{array}$ & $\begin{array}{l}\text { Homens negros que } \\
\text { frequentam instituições } \\
\text { de ensino superior. }\end{array}$ & $\begin{array}{l}\text { Examinou-se a associação entre o } \\
\text { consumo de álcool e as condições de } \\
\text { saúde mental e buscou-se determinar as } \\
\text { diferenças entre homens negros em } \\
\text { instituições predominantemente brancas } \\
\text { (PWIs) em comparação com aqueles } \\
\text { que frequentavam instituições } \\
\text { predominantemente minoritárias. }\end{array}$ & $\begin{array}{l}\text { Homens negros que frequentavam uma PWI } \\
\text { relataram níveis significativamente maiores de } \\
\text { consumo de álcool e significativamente mais } \\
\text { transtornos mentais. Participar de uma } \\
\text { instituição que atende majoritariamente a } \\
\text { minorias foi associada a menos transtornos } \\
\text { mentais entre os homens negros. }\end{array}$ \\
\hline $\begin{array}{l}\text { Análise da Qualidade e } \\
\text { Estilo de Vida entre } \\
\text { Acadêmicos de Medicina } \\
\text { de uma Instituição do } \\
\text { Norte do Paraná. }\end{array}$ & $\begin{array}{l}\text { Universitários do } \\
\text { primeiro ao sexto ano do } \\
\text { curso de Medicina, a } \\
\text { maioria com idade entre } \\
21 \text { e } 25 \text { anos, sexo } \\
\text { feminino, cor/raça } \\
\text { branca, solteiros, } \\
\text { estudaram em escola } \\
\text { privada no ensino médio } \\
\text { e moram sozinhos. }\end{array}$ & $\begin{array}{l}\text { Empregou-se } \\
\begin{array}{l}\text { autoaplicável } \\
\text { "Questionário de Estilo de Vida } \\
\text { denominado }\end{array} \\
\text { Fantástico", que considera o } \\
\text { comportamento dos indivíduos e seus } \\
\text { resultados permitem determinar a } \\
\text { associação entre estilo de vida e saúde. } \\
\text { A soma de todos os pontos permite } \\
\text { chegar a um escore total que classifica } \\
\text { os indivíduos em cinco categorias. }\end{array}$ & $\begin{array}{l}\text { Menos da metade dos estudantes apresentaram } \\
\text { diagnóstico de depressão ou outra patologia } \\
\text { crônica psiquiátrica. Grande parte dos alunos } \\
\text { não dorme bem e não se sente descansada. } \\
\text { Observou-se alta prevalência de estudantes } \\
\text { que estão satisfeitos com seus trabalhos ou } \\
\text { funções. A maioria referiu não ter fumado no } \\
\text { último ano e nunca ter usado drogas como } \\
\text { maconha e cocaína, enquanto } 81 \% \text { relataram } \\
\text { uma ingestão média de álcool por semana de } \\
\text { zero a sete doses. }\end{array}$ \\
\hline $\begin{array}{l}\text { Psychiatric Symptoms } \\
\text { and Diagnoses Among } \\
\text { U.S. College Students: A } \\
\text { Comparison by Race and } \\
\text { Ethnicity. }\end{array}$ & $\begin{array}{l}\text { Alunos de } 108 \text { faculdades } \\
\text { ou universidades nos } \\
\text { Estados Unidos com pelo } \\
\text { menos } 18 \text { anos de idade. }\end{array}$ & $\begin{array}{l}\text { Houve taxa de resposta de } 18 \% \text { para } \\
\text { pesquisa online e de } 83 \% \text { em papel, e } \\
\text { cada instituição determinou o formato } \\
\text { de questionário, que averiguava } \\
\text { sintomas psiquiátricos, diagnósticos e } \\
\text { características sociodemográficas. }\end{array}$ & $\begin{array}{l}\text { As taxas de sintomas psiquiátricos e } \\
\text { diagnósticos relatados no ano anterior foram } \\
\text { altas, independentemente da raça ou etnia. Em } \\
\text { comparação com brancos, negros e hispânicos } \\
\text { endossaram taxas mais baixas de diagnósticos } \\
\text { psiquiátricos, mas tiveram taxas semelhantes } \\
\text { de tentativas de suicídio no ano anterior. }\end{array}$ \\
\hline $\begin{array}{l}\text { Differences in Hope, } \\
\text { Core Self-Evaluations, } \\
\text { Emotional Well-Being, } \\
\text { and Health Risk } \\
\text { Behaviors in Freshman } \\
\text { University Students. }\end{array}$ & $\begin{array}{l}\text { Calouros universitários } \\
\text { com idades entre } 18-21 .\end{array}$ & $\begin{array}{l}\text { Um escore de comportamento de risco à } \\
\text { saúde, com pontuações variando de } 3 \text { a } \\
9 \text {, foi calculado criando categorias de } \\
\text { risco para uso de drogas, uso de álcool e } \\
\text { comportamento sexual de risco. }\end{array}$ & $\begin{array}{l}\text { Havia diferenças raciais na esperança, bem- } \\
\text { estar emocional e comportamentos de risco à } \\
\text { saúde. Enfermeiros e pesquisadores devem } \\
\text { considerar as diferenças raciais e de gênero ao } \\
\text { projetar ou implementar intervenções. }\end{array}$ \\
\hline $\begin{array}{l}\text { Addressing Mental } \\
\text { Health } \\
\text { Perspectives of African } \\
\text { Americans Living in the } \\
\text { Rural South. }\end{array}$ & $\begin{array}{l}\text { Prestadores de cuidados } \\
\text { primários, comunidade } \\
\text { religiosa, estudantes } \\
\text { universitários r e } \\
\text { administradores, e } \\
\text { indivíduos que vivem } \\
\text { com distúrbios mentais. }\end{array}$ & $\begin{array}{l}\text { Um guia de entrevista semiestruturado } \\
\text { foi usado para eliciar perspectivas de } \\
\text { saúde mental, tratamento de saúde } \\
\text { mental e maneiras de melhorar a saúde } \\
\text { mental nas comunidades rurais afro- } \\
\text { americanas. }\end{array}$ & $\begin{array}{l}\text { Ambientes de vida estressantes e crenças } \\
\text { comunitárias impactaram não apenas as } \\
\text { percepções de saúde mental, mas também } \\
\text { contribuíram para as barreiras que impedem a } \\
\text { busca por suporte acerca de saúde mental. Os } \\
\text { participantes também identificaram estratégias } \\
\text { em nível de comunidade que podem ser } \\
\text { utilizadas para melhorar o bem-estar } \\
\text { emocional em comunidades rurais afro- } \\
\text { americanas. }\end{array}$ \\
\hline $\begin{array}{l}\text { Mental Health Outcomes } \\
\text { of Discrimination among } \\
\text { College Students on a } \\
\text { Predominately White } \\
\text { Campus: A Prospective } \\
\text { Study. }\end{array}$ & $\begin{array}{l}\text { Utilizou-se dados de uma } \\
\text { amostra racialmente } \\
\text { diversa de estudantes } \\
\text { universitários em uma } \\
\text { universidade } \\
\text { majoritariamente branca. }\end{array}$ & $\begin{array}{l}\text { Os efeitos da saúde mental examinados } \\
\text { incluíram cinco escalas: (1) raiva, (2) } \\
\text { ansiedade, (3) solidão, (4) sintomas } \\
\text { depressivos e (5) afeto positivo. Os } \\
\text { itens para cada uma das escalas foram } \\
\text { retirados do National Institutes of } \\
\text { Health (NIH) Toolbox e the Center for } \\
\text { Epidemiologic Studies Depression } \\
\text { Scale (CES-D). Três variáveis } \\
\text { preditoras principais são usadas na } \\
\text { análise: (1) discriminação interpessoal, }\end{array}$ & $\begin{array}{l}\text { Os resultados mostram que eventos sociais } \\
\text { relacionados à discriminação interpessoal } \\
\text { predizem maior raiva, ansiedade, sintomas } \\
\text { depressivos e solidão autorreferidos, tanto } \\
\text { diariamente como em média ao longo do } \\
\text { tempo. O racismo vicário do dia a dia foi } \\
\text { associado ao aumento dos sintomas de } \\
\text { ansiedade. Em contraste, a ruminação da } \\
\text { discriminação não foi associada a resultados } \\
\text { negativos de saúde mental. }\end{array}$ \\
\hline
\end{tabular}




\begin{tabular}{|c|c|c|c|}
\hline & & (2) racismo vicário e (3) ruminação. & \\
\hline $\begin{array}{l}\text { Racial/ethnic variation in } \\
\text { mental health correlates } \\
\text { of substance use among } \\
\text { college students. }\end{array}$ & $\begin{array}{l}\text { Uma amostra } \\
\text { probabilística de alunos } \\
\text { em duas universidades da } \\
\text { Califórnia. }\end{array}$ & $\begin{array}{l}\text { Investigou-se indicadores de saúde } \\
\text { mental, uso de substâncias e suas } \\
\text { relações, por raça/etnia. Os participantes } \\
\text { relataram sua frequência de uso de } \\
\text { substâncias e avaliaram sua experiência } \\
\text { com indicadores de saúde mental. }\end{array}$ & $\begin{array}{l}\text { Análise de variância unilateral (ANOVA), } \\
\text { testes de qui-quadrado e modelos de regressão } \\
\text { multivariados censurados foram estimados } \\
\text { para examinar quais indicadores de saúde } \\
\text { mental estavam associados a cada forma de } \\
\text { uso de substância por raça/etnia. Os resultados } \\
\text { mostraram diferenças na prevalência do uso de } \\
\text { substâncias e saúde mental por raça/etnia. } \\
\text { Alunos brancos demonstram prevalência mais } \\
\text { alta para todas as formas de uso de } \\
\text { substâncias. }\end{array}$ \\
\hline $\begin{array}{l}\text { Promising Results from } \\
\text { the Use of a Korean } \\
\text { Drama to Address } \\
\text { Knowledge, Attitudes, } \\
\text { and Behaviors on School } \\
\text { Bullying and Mental } \\
\text { Health among Asian } \\
\text { American College-Aged } \\
\text { Students. }\end{array}$ & $\begin{array}{l}\text { Amostra de estudantes } \\
\text { universitários. }\end{array}$ & $\begin{array}{l}\text { Examinou-se o conhecimento, as } \\
\text { atitudes e os comportamentos a respeito } \\
\text { do bullying escolar (KAB) melhoraram } \\
\text { após assistir-se a um K-Drama } \\
\text { retratando o bullying na escola e } \\
\text { problemas de saúde mental. Perguntou- } \\
\text { se aos participantes sobre suas } \\
\text { perspectivas de usar um K-Drama como } \\
\text { intervenção. Pré-/pós-testes sobre KAB } \\
\text { foram realizados. Entrevistas foram } \\
\text { usadas para entender suas experiências } \\
\text { com K-Dramas. }\end{array}$ & $\begin{array}{l}\text { Muitos relataram experiências com ansiedade } \\
(67,8 \%) \text {, depressão }(38,1 \%) \text { e experiência de } \\
\text { vítima de bullying escolar }(40,8 \%) \text {. As } \\
\text { pontuações pós-teste revelaram diferenças } \\
\text { significativas no conhecimento sobre a } \\
\text { maioria das variáveis de bullying escolar (por } \\
\text { exemplo, vítima; testemunha) e questões de } \\
\text { saúde mental. Os participantes relataram que } \\
\text { "amam" o drama, sentiram uma conexão } \\
\text { emocional e pensaram que K-Dramas podem } \\
\text { ser uma ferramenta educacional. }\end{array}$ \\
\hline $\begin{array}{l}\text { Bicultural Identity } \\
\text { Harmony and American } \\
\text { Identity Are Associated } \\
\text { With Positive Mental } \\
\text { Health in U.S. Racial and } \\
\text { Ethnic Minority } \\
\text { Immigrants. Cultural } \\
\text { Diversity and Ethnic } \\
\text { Minority Psychology. }\end{array}$ &  & $\begin{array}{l}\text { Os participantes responderam a um } \\
\text { questionário composto por perguntas } \\
\text { relacionadas às suas identidades étnicas } \\
\text { e americanas, o grau em que essas } \\
\text { identidades são compatíveis (isto é, } \\
\text { Integração da Identidade Bicultural; } \\
\text { BII) e a presença de sintomas de } \\
\text { depressão e ansiedade. }\end{array}$ & $\begin{array}{l}\text { As análises mostraram associações negativas } \\
\text { significativas entre a identidade americana e a } \\
\text { harmonia cultural BII com os sintomas de } \\
\text { depressão. A harmonia cultural BII também } \\
\text { foi negativamente associada aos sintomas de } \\
\text { ansiedade. A harmonia cultural BII mediou as } \\
\text { relações entre a identidade americana e os } \\
\text { sintomas de depressão e ansiedade. Tanto para } \\
\text { a harmonia cultural quanto para a mescla } \\
\text { cultural e identidade americana, hispânicos } \\
\text { pontuaram significativamente mais alto que } \\
\text { negros. }\end{array}$ \\
\hline $\begin{array}{l}\text { Do Dimensions of Ethnic } \\
\text { Identity Mediate the } \\
\text { Association Between } \\
\text { Perceived Ethnic Group } \\
\text { Discrimination and } \\
\text { Depressive Symptoms? }\end{array}$ & $\begin{array}{l}\text { Estudantes universitários } \\
\text { de minorias étnicas (de } \\
18 \text { a } 30 \text { anos; } 37 \% \\
\text { negros, } 63 \% \text { latinos). } \\
76 \% \text { eram do sexo } \\
\text { feminino. Em média, os } \\
\text { participantes tinham } 20 \\
\text { anos. }\end{array}$ & $\begin{array}{l}\text { Os dados foram coletados por meio de } \\
\text { uma pesquisa online. A discriminação } \\
\text { de grupo étnico percebida foi avaliada a } \\
\text { Escala de Experiência Étnica, a } \\
\text { identidade étnica foi medida usando a } \\
\text { Escala de Identidade Étnica e os } \\
\text { sintomas depressivos foram medidos } \\
\text { usando o Center for Epidemiologic } \\
\text { Studies Depression Scale. }\end{array}$ & $\begin{array}{l}\text { A percepção de discriminação foi associada } \\
\text { positivamente a sintomas depressivos em } \\
\text { estudantes de ambos os grupos étnicos. A } \\
\text { relação entre a percepção de discriminação e } \\
\text { sintomas depressivos foi mediada pela } \\
\text { afirmação da identidade étnica para estudantes } \\
\text { latinos, mas não para estudantes negros. }\end{array}$ \\
\hline $\begin{array}{l}\text { Risk factors and } \\
\text { outcomes of chronic } \\
\text { sexual harassment } \\
\text { during the transition to } \\
\text { college: Examination of a } \\
\text { two-part growth mixture } \\
\text { model. }\end{array}$ & $\begin{array}{l}\text { Alunos universitários } \\
\text { calouros }(58 \% \text { mulheres; } \\
54 \% \text { brancos e } 8,1 \% \\
\text { afro-americanos). }\end{array}$ & $\begin{array}{l}\text { A amostra completou uma pesquisa em } \\
\text { cinco pontos no tempo. Além de relatar } \\
\text { gênero, raça e orientação sexual, } \\
\text { completaram medidas de assédio } \\
\text { sexual, ansiedade, depressão, consumo } \\
\text { excessivo de álcool e uso de maconha. }\end{array}$ & $\begin{array}{l}\text { Ser branco, afro-americano ou } \\
\text { outro/multirracial e de uma minoria sexual foi } \\
\text { associado a uma maior probabilidade de ser } \\
\text { classificado na classe Crônica (vs. } \\
\text { Infrequente) de assédio sexual. Em relação aos } \\
\text { brancos, ser afro-americano e hispânico/latino } \\
\text { predisse diretamente depressão elevada. }\end{array}$ \\
\hline $\begin{array}{l}\text { A delay-discounting } \\
\text { measure of preference } \\
\text { for racial/ethnic } \\
\text { matching in } \\
\text { psychotherapy. }\end{array}$ & $\begin{array}{l}\text { Uma amostra de alunos } \\
\text { de minorias } \\
\text { raciais/étnicas de } 2 \\
\text { universidades e uma } \\
\text { amostra nacional de } \\
\text { clientes (incluindo } 20 \\
\text { universitários) } \\
\text { psicoterapia. A maioria } \\
\text { dos alunos era do sexo } \\
\text { feminino (68,1\%), com } \\
\text { média de idade de } 21 \\
\text { anos. Afro-americanos } \\
\text { correspondiam a } 28,4 \% \\
\text { dos estudantes, além de } \\
\text { hispânicos/latinos } \\
\text { (37,8\%), etc. }\end{array}$ & $\begin{array}{l}\text { Empregando um método delay- } \\
\text { discounting, os sujeitos da pesquisa } \\
\text { indicaram sua preferência por } 4 \\
\text { métodos diferentes para abordar } \\
\text { variáveis culturalmente relacionadas em } \\
\text { psicoterapia, incluindo (a) desejo de } \\
\text { trabalhar com um terapeuta cuja } \\
\text { raça/etnia corresponda à sua, (b) com } \\
\text { um terapeuta com alto nível de } \\
\text { treinamento e experiência multicultural, } \\
\text { (c) receber um tratamento adaptado } \\
\text { culturalmente, e (d) um terapeuta que } \\
\text { também é de minoria racial/étnica, mas } \\
\text { não a mesma que a sua. }\end{array}$ & $\begin{array}{l}\text { Os participantes estavam dispostos a fazer } \\
\text { sacrifícios significativos na eficácia do } \\
\text { tratamento para receber cada uma das } \\
\text { variáveis testadas. Em ambas as amostras, as } \\
\text { preferências foram significativamente mais } \\
\text { fortes para o treinamento/experiência } \\
\text { multicultural do terapeuta e o uso de } \\
\text { tratamentos adaptados culturalmente em } \\
\text { comparação com a correspondência } \\
\text { racial/étnica e pareamento de minoria } \\
\text { racial/étnica. Os resultados deste estudo têm } \\
\text { implicações importantes para acomodação } \\
\text { preferencial em psicoterapia com indivíduos } \\
\text { de minorias raciais/étnicas. }\end{array}$ \\
\hline
\end{tabular}


Fonte: Protocolo de pesquisa.

Como pode ser observado na Tabela 2, 8 estudos eram transversais quantitativos. Além desses, há 1 meta-síntese interpretativa qualitativa; 1 estudo transversal o qual fez uso de uma entrevista semiestruturada, ou seja, com aspectos tanto quantitativos quanto qualitativos; e 3 estudos longitudinais, sendo que um empregou uma mídia audiovisual como intervenção. Além disso, todos os transversais usaram questionários para a realização da pesquisa, além de outros métodos próprios de cada pesquisa, conforme explicitado. Apenas uma referência focava especificamente em alunos de Medicina.

\section{Discussão}

A questão de maior destaque durante o desenvolvimento desta pesquisa foi a escassez de estudos acerca da saúde mental de estudantes negros no Ensino Superior. À primeira vista, parece ser uma temática interessante para direcionar estudos, não obstante, na prática, há uma enorme ausência de artigos sobre saúde mental na Educação Superior cujas variáveis incluem raça. Mesmo quando incluído, percebe-se que não existe foco ou aprofundamento na saúde mental dos estudantes de raça negra, principalmente visando investigar os impactos do racismo.

$\mathrm{Na}$ base de dados SciElo, em que grande parte dos estudos são de origem brasileira, ainda que usando diferentes palavras-chave e diferentes combinações para pesquisa, notou-se a escassez de artigos sobre o assunto que esta revisão sistemática propõe-se a averiguar; algo no mínimo curioso, uma vez que a maior parte da população brasileira é negra. No PubMed, por sua vez, em que existe abundância de artigos sobre os mais variados temas, apesar de a busca ter resultado em uma quantidade significativa de trabalhos, a maioria visava indivíduos asiáticos amarelos ou latinos. Dentre os poucos que consideravam a raça negra, todos eram de origem estadunidense.

No artigo de Buhrer et al (2019), exemplificando, a respeito da qualidade e estilo de vida entre acadêmicos de medicina, embora a metodologia inclua a variável raça, é atestado que "a população foi constituída essencialmente por indivíduos que se auto classificaram como de raça branca (86,63\%)" e explicam essa prevalência por tratar-se de uma faculdade no Paraná, na Região Sul do Brasil, marcada pela presença da colonização europeia. De fato, apenas 9 em cada 100 estudantes em cursos de Medicina no Paraná são negros (Portal Bem Paraná, 2019). Dessa maneira, a negligência perante a população preta prontamente é verificada pela reduzida participação do grupo em um curso de grande prestígio como Medicina, ainda que um pouco mais de um terço da população do Paraná seja formada por negros (Portal G1, 2019).

Barnett et al. (2019), de uma perspectiva estadunidense, reporta que estudantes pretos em instituições predominantemente brancas, muitas vezes, sentem-se pressionados a se misturar à realidade cultural dominante para se encaixar. Percebe-se, desse modo, uma tendência semelhante à descrita por Frantz Fanon: "Que quer o homem? Que quer o homem negro? [...] O negro quer ser branco," citado por Bernardino-Costa (2016). Segundo o mesmo estudo de Barnett, campi universitários são um microcosmo da sociedade, com a presença dos mesmos estereótipos e baixas expectativas para pretos. Aproximadamente $12 \%$ a $50 \%$ dos estudantes universitários atendem aos critérios para um ou mais transtornos mentais. Os afro-americanos não só precisam lidar com a transição da adolescência para a vida adulta, que já é um estressor, como também com microagressões raciais; assim, geralmente, têm saúde mais agravada, com maiores taxas de doenças cardiovasculares e depressão.

Com enfoque em universitários calouros, Griggs e Sybil (2019) exploram o bem-estar emocional do grupo, autoavaliação, esperança, comportamentos de risco e desempenho acadêmico; usando como uma das variáveis a raça. Em relação à esperança, houve diferenças raciais. Os autores salientam a necessidade de outras pesquisas com maiores amostras de grupos não-brancos, uma vez que apenas 3,5\% dos participantes eram afro-americanos. Nos resultados da pesquisa, 
contrariando as demais aqui apresentadas, afro-americanos relataram o maior bem-estar emocional, seguido por hispânicos, caucasianos, asiáticos e outras raças; "no entanto, não se sabe se esta prevalência mais baixa está relacionada à acesso mais pobre a diagnóstico e tratamento neste grupo racial". Brancos apresentaram os maiores índices de esperança e, simultaneamente, maiores níveis de comportamentos de risco à saúde, em convergência com outros estudos que apontam maiores taxas de uso de substâncias por este grupo racial. É especificado, contudo, que as diferenças raciais devem ser interpretadas com precaução devido às limitações do estudo e dos instrumentos aplicados.

Em contraposição, Chen et al. (2019) compararam sintomas e diagnósticos psiquiátricos por raça e etnia em larga escala, abrangendo 108 universidades e mais de 67 mil participantes, visando atualizar o cenário de pesquisa sobre a saúde mental de grupos minoritários. Alunos hispânicos, pretos e asiáticos/das ilhas do Pacífico apresentaram menor probabilidade de relatar qualquer sintoma ou diagnóstico psiquiátrico no ano anterior ao questionário em comparação com brancos. Todavia, nesta amostra, mais de 43 mil eram brancos e apenas 3 mil eram pretos, número inferior à quantidade de hispânicos e asiáticos; mais uma vez, tal qual o estudo citado anteriormente, evidenciando uma discrepância. Além disso, dentre os participantes pretos, mais de $70 \%$ eram mulheres cisgênero e quase $90 \%$ era heterossexual. Ainda assim, o artigo ressalta que está havendo aumento de diversidade dentro das universidades, bem como o aumento de transtornos mentais.

O mesmo estudo realça que há grande risco de minorias raciais enfrentarem transtornos mentais não detectados. A pesquisa apontou que, embora estudantes pretos e hispânicos tenham menor probabilidade de reportar sintomas de transtorno mental que brancos, os seus índices de tentativas de suicídio no ano anterior eram significativamente mais elevados.

Barry et al. (2016), por sua vez, propõe-se a examinar o uso de álcool entre homens pretos, comparando em instituições predominantemente brancas com instituições em que predominam minorias. Como a maior parte dos estudos nesta revisão, já no abstract é ressaltado a escassez de pesquisas cuja amostra envolva a população negra, particularmente homens, o que é preocupante devido à sua susceptibilidade a problemas relacionados à saúde mental; em relação a ingestão de bebidas alcóolicas, também o fazem mais que mulheres negras. Homens pretos que frequentavam uma instituição predominantemente branca relataram níveis consideravelmente maiores de consumo de álcool - conquanto não tinham índices tão altos de ingestão alcoólica quanto brancos, assim como relatam Griggs e Sybil (2019) - e mais transtornos mentais.

Similarmente, Sumstine et al. (2017) encarrega-se de averiguar indicadores de saúde mental, uso de substâncias e sua relação com raça e etnia. Contudo, nesta análise, os participantes pretos são postos na associação “All other", incluindo afroamericanos, nativos, birraciais/multinacionais e outros; o que ocorreu devido a pequenas quantidades desses grupos. Mais uma vez, nota-se a reduzida participação de pretos nas universidades. Certamente, não obstante, é necessário levar em consideração a distribuição demográfica. De acordo com os dados oficiais, na Califórnia, onde o estudo aconteceu, a população negra é apenas 6,5\% (CENSUS, 2021). Observa-se, dessa forma, que isto pode ser um obstáculo, em pesquisas estadunidenses, para analisar a população negra dentro de um contexto geral, comparando a outros agrupamentos raciais. Assim, pondera-se a carência de literaturas com enfoque direto em estudantes pretos.

Além disso, ressalta-se a interpretação peculiar de raça/etnia para estadunidenses, tendo em vista que "latino" entra como um grupo ético, ainda que, na verdade, sejam pessoas provenientes da América Latina, que podem ser brancas, negras, amarelas, etc. Por fim, ainda no estudo de Sumstine et al. (2017), enfatiza-se, outra vez, resultados sublinhando que brancos demonstraram maior prevalência no uso de todas as substâncias.

O estudo de Barnett et al. (2019) é um dos mais interessantes. Investiga-se tanto percepções de saúde física quanto mental (abrangendo estresse, confiança e espiritualidade) a partir de uma pesquisa qualitativa, em que os autores são usados como instrumentos, exigindo, portanto, uma discussão de vieses potenciais. Na descrição feita, verifica-se que quatro dos cinco são afro-americanos. Dentre a amostra, alguns alunos compartilharam que comer era uma de suas principais maneiras de enfrentamento para lidar com estressores da vida diária. Até mesmo o cabelo aparece como fator de interferência na saúde 
mental, visto que é muitíssimo relevante para as mulheres afro-americanas e a pressão da manutenção pode causar estresse prejudicial à saúde.

"A harmonia de identidade bicultural e a identidade americana estão associadas a saúde mental positiva em imigrantes de minorias raciais e étnicas nos EUA" é um título que já diz muito por si só, trata-se do trabalho de Tikhonov et al. (2019); como sugerido, examina-se a associação de identidade étnica e estadunidense com depressão e ansiedade e se esta relação é mediada pela integração de identidade bicultural. 19.8\% dos participantes eram imigrantes negros universitários. Tanto para a harmonia cultural quanto para a mescla cultural, os participantes hispânicos pontuaram significativamente mais alto que negros, bem como negros pontuaram menos que hispânicos para identidade americana. Concluiu-se que a compatibilidade entre as identidades étnicas com a identidade nacional estadunidense é aparentemente importante para saúde mental entre imigrantes de minorias raciais e étnicas, algo entendível atendendo ao pressuposto de que a migração e adaptação a uma nova realidade local e sociocultural são estressores predisponentes de transtornos mentais, especialmente para minorias em que o racismo é uma pauta.

Mais uma vez, o aspecto "identidade" e "pertencimento" entram em questão para universitários negros: a violência que é não se identificar e não se sentir pertencente à realidade e/ou local em que se está. Os próprios autores apontam, para mais, que pesquisas longitudinais ajudariam a avaliar a causalidade. Independentemente de ser uma pesquisa transversal, o texto de Brittian et al. (2015) é complementar ao citado anteriormente e põe que a identidade étnica pode promover ajustamento psicológico no contexto de experiências discriminatórias, sendo as dimensões da identidade étnica: exploração, resolução e afirmação. Nele, $37 \%$ dos participantes são negros e $63 \%$ são latinos - outra vez, vislumbra-se a concepção de etnia para estadunidenses, visto que há, exemplificativamente, latinos negros e brancos. Os participantes identificaram seu grupo étnico, sendo as opções: Negro/Afro-americano, Caucasiano/Branco, Asiático/ das ilhas do Pacífico, Latino/ Hispânico, do Oriente Médio/Árabe e Pessoa-de-cor-da-África do Sul/Malaio do Cabo. Entre os alunos negros, 85\% nasceram nos Estados Unidos e 15\% nasceram fora dos Estados Unidos.

A relação entre a percepção de discriminação e sintomas depressivos foi mediada pela afirmação da identidade étnica para estudantes latinos, mas não para estudantes negros. Os autores justificam que, provavelmente, os estudantes negros possam ter atribuído a discriminação a forças institucionais ou sociais, enquanto latinos podem ter atribuído à língua e à imigração e internalizado essas experiências. Além disso, "análises preliminares indicaram que estudantes negros relataram níveis mais altos de percepção da discriminação e exploração da sua identidade étnica." Ainda, em média, latinos relataram níveis maiores de afirmação da identidade étnica. De qualquer forma, a percepção de estar sofrendo discriminação foi associada positivamente a sintomas depressivos em estudantes de ambos os grupos. Os autores descrevem também que "atitudes sociais e barreiras para grupos étnicos, e não apenas experiências pessoais com discriminação no campus da faculdade, são fatores de risco notáveis para problemas de saúde mental."

Ademais, devido à história da escravatura nos Estados Unidos e à Guerra Civil, o Sul carrega marcas ainda mais profundas provocadas pelo racismo do que o resto do país (não significa que as outras regiões estejam livres dessa violência), $o$ que estende-se à sua área rural. "Afro-americanos rurais são desproporcionalmente afetados por estressores sociais que os colocam em risco para doenças psiquiátricas", por isso, o estudo de Haynes et al. (2017) procurou entender as barreiras ao tratamento desse povo, a fim de conceber culturalmente abordagens de tratamento aceitáveis. Para isso, um dos grupos estudados foi de estudantes universitários. Os resultados denotaram que ambientes estressantes e crenças comunitárias têm impacto sobre a percepção de saúde mental e interferem na busca por ajuda. Entre as estratégias para elevar o bem-estar emocional, sugeriu-se, por exemplo, a promoção de suporte social e conscientização sobre saúde mental para o público. Entretanto, embora este seja um dos poucos artigos que foca exclusivamente na população negra, os resultados não separam as respostas dos universitários dos demais grupos, ou seja, não é possível saber quais foram as respostas deste grupo 
singularmente.

Apesar de não incorporar a população preta, Park et al. (2020) apresenta uma proposta interessante à perspectiva de cuidado à saúde mental de minorias raciais. "Os programas de televisão de drama coreanos (K-Dramas) podem ser uma abordagem inovadora para melhorar o conhecimento, as atitudes e os comportamentos sobre o bullying". Dessa maneira, uma amostra de estudantes universitários asiáticos-americanos assistiu a um K-Drama retratando o bullying na escola e problemas de saúde mental. Foram realizados pré e pós-testes sobre KAB (knowledge, attitudes, and behaviors ou conhecimento, atitudes e comportamento) acerca de bullying. Os participantes expuseram que "amaram" o drama, sentiram conexão emocional e concordaram que K-Dramas podem ser uma ferramenta educacional de auxílio à saúde das massas. Logo, o estudo introduz mídias como uma opção viável de conscientização para ajudar no combate à discriminação.

Swift et al. trazem uma abordagem muito significante com uma amostra de alunos de minorias raciais/étnicas e uma amostra nacional de clientes (incluindo 20 universitários) de psicoterapia. A maioria dos alunos era do sexo feminino $(68,1 \%)$, com média de idade de 21 anos. Afro-americanos correspondiam a 28,4\% dos estudantes, além de hispânicos/latinos (37,8\%), etc. Os sujeitos da pesquisa indicaram sua preferência por 4 métodos diferentes para abordar variáveis culturais na psicoterapia, incluindo (a) desejo de trabalhar com um terapeuta cuja raça/etnia corresponda à sua, (b) com um terapeuta com alto nível de treinamento e experiência multicultural, (c) receber um tratamento adaptado culturalmente, e (d) um terapeuta que também é de minoria racial/étnica, mas não a mesma que o participante. Em ambas as amostras, as preferências foram, de modo considerável, mais fortes para o treinamento/experiência multicultural do terapeuta e o uso de tratamentos adaptados culturalmente em comparação à correspondência racial/étnica e pareamento de minoria racial/étnica. Entre estudantes que relataram o uso anterior de psicoterapia, a menor porcentagem era afro-americana (14,9\%).

$\mathrm{O}$ assunto trabalhado aqui mediante diversos artigos está em concordância com a teoria do estresse de minoria (EM), em que minorias sociais vivenciam estressores específicos devido à discriminação que são adicionais aos estressores cotidianos, sucedendo maior vulnerabilidade social. O estresse pode ser, para mais, potencializado fazendo-se parte de múltiplas minorias. Aspirando pesquisas posteriores, é pertinente avaliar a saúde mental de grupos com indivíduos submetidos a diferentes tipos de preconceito, tal como pessoas negras pertencentes a minorias sexuais. "Fatores individuais e do meio podem funcionar como fatores de risco e/ou de proteção no comprometimento da saúde mental de pessoas LGB", a revisão narrativa de Paveltchuk e Borsa (2020) pontua.

As propensões a cada gênero também não podem ser ignoradas. McGinley et al. (2016), com uma amostra de 58\% mulheres e 8,1\% afro-americanos em um estudo quanto a assédio sexual, saúde mental e consumo de substâncias, discorrem que ser branco, afro-americano ou outro/multirracial, mulher, e de uma minoria sexual foi associado a maior probabilidade de estar na classe Crônica (vs. Infrequente) de assédio sexual. Em relação aos brancos, ser afro-americano e hispânico/latino predisse diretamente depressão elevada no quarto período de colheita de dados da pesquisa longitudinal. Outro achado é o de que aqueles que desistiram do estudo eram substancialmente mais predispostos a ser afro-americanos, minoria sexual, deprimidos, envolver-se em mais bebedeiras e experienciar mais assédio sexual do que quem forneceu dados até o fim.

As limitações desta revisão sistemática concernem à complexidade do assunto, existindo, porém, poucas divergências em relação aos resultados. Vale ressaltar que o tema em debate é pouco comentado, sobretudo em território nacional, e por isso é difícil confrontar seus dados em nível nacional (Brasil) e global, posto que mais de $90 \%$ dos artigos descritos surgem da nacionalidade e cultura estadunidense. Dessa forma, para futuras revisões, recomenda-se o emprego de trabalhos acerca de diferentes nações ou salientando a brasileira para que a comparação de dados seja mais sublime. Além disso, utilizaram-se somente artigos disponíveis online nas bases de dados citadas, o que limita a literatura analisada. Ademais, apesar de possuir como amostra estudantes negros universitários, alguns estudos não tomam como foco o ambiente dentro do espaço físico da universidade. Nesta revisão sistemática, é válido frisar, não houve aprofundamento na comparação de idade entre, por 
exemplo, alunos negros de 18 anos e de 60 anos, em estágios diferentes da vida que englobam diferentes estressores; uma variável interessante para discorrer futuramente. Para a população brasileira, nesta pesquisa encontra-se particularmente o que podemos aprender doravante com o cenário estadunidense.

\section{Conclusão}

O presente estudo possui lacunas referentes à escassa literatura cujo enfoque inclui a variável raça/cor da pele ou que abranjam o tema proposto, sobretudo às que concernem ao território nacional. Por conseguinte, elucida a negligência enfrentada por parte das pessoas não-brancas.

É fundamental, também, que a comunidade científica realize pesquisas com amostras maiores da população negra. Da mesma forma, que sejam separados os agrupamentos raciais e não analisados em um contexto geral ou grupo único, visto que cada um contém determinantes sociais de saúde distintos. Assim, tendo em vista uma investigação mais precisa e minuciosa da saúde mental dos estudantes negros do nível superior, para formulação de medidas eficazes no enfrentamento das implicações do racismo verificadas nesse grupo social.

Não obstante às limitações na pesquisa desta temática, foi possível evidenciar consonância quanto aos resultados encontrados. De tal forma que se faz notória a vulnerabilidade ao qual os estudantes pretos, neste estudo restritos ao nível superior de ensino, estão submetidos, aumentando expressivamente os riscos de desenvolvimento de algum agravo na saúde mental. Desse modo, o ambiente universitário assume o reflexo das relações de poder vivenciadas na sociedade, abrangendo todas as formas de discriminação e de preconceito, as quais repercutem na qualidade de vida dos grupos oprimidos.

\section{Referências}

Barnett, T. M., McFarland, A., Miller, J. W., Lowe, V. \& Hatcher, S. S. (2019) Physical and Mental Health Experiences among African American College Students, Soc Work Public Health. 34(2), 145-157. http://doi.org/10.1080/19371918.2019.1575308.

Barry, A. E. Jackson, Z. Watkins, D. C. Goodwill, J. R. \& Hunte, H. E R. (2017) Alcohol Use and Mental Health Conditions Among Black College Males: Do Those Attending Postsecondary Minority Institutions Fare Better Than Those at Primarily White Institutions? American Journal of Men's Health, 11(4), 962968. https://doi.org/10.1177/1557988316674840.

Batista, W. M. (2018) A inferiorização dos negros a partir do racismo estrutural. Rev. Direito Práx., 9(4), 2581-2589. https://doi.org/10.1590/2179$8966 / 2018 / 36867$

Bernardino-Costa, J. (206) A prece de Frantz Fanon: Oh, meu corpo, faça sempre de mim um homem que questiona! Civitas, Rev. Ciênc. Soc. 16(3), 504-521.

Braga, P. R. S., \& Milani, C. R. S. (2019). Direitos humanos e política externa no Brasil e na África do Sul: o mito da democracia racial, o apartheid e as narrativas da redemocratização. Rev. Bras. Ciênc. Polit. 29, 7-44. https://doi.org/10.1590/0103-335220192901.

BRASIL, Ministério da Saúde, Secretaria de Gestão Estratégica e Participativa. (2016). Temático Saúde da População Negra. 7.

Brittian, A. S., Kim, S. Y., Armenta, B. E., Lee, R. M., Umaña-Taylor, A. J., Schwartz, S. J., Villalta, I. K., Zamboanga, B. L., Weisskirch, R. S., Juang, L. P., Castillo, L. G., \& Hudson, M. (2015). Do dimensions of ethnic identity mediate the association between perceived ethnic group discrimination and depressive symptoms? Cultural diversity \& ethnic minority psychology 21(1), 41-53. https://doi.org/10.1037/a0037531.

Bronoski, B. (2020). Pouco mais de um terço da população do Paraná é formada por negros, e governo estadual não tem Políticas Públicas específicas. https://g1.globo.com/pr/parana/noticia/2020/07/21/pouco-mais-de-um-terco-da-populacao-do-parana-e-formada-por-negros-e-governo-estadual-nao-tempoliticas-publicas-especificas.ghtml

Bührer, B. E., Tomiyoshi, A. C., Furtado, M. D., \& Nishida, F. S. (2019). Análise da Qualidade e Estilo de Vida entre Acadêmicos de Medicina de uma Instituição do Norte do Paraná. Rev. bras. educ. med., 43(1), 39-46. https://doi.org/10.1590/1981-52712015v43n1RB20170143.

CENSUS - United States Census Bureau (2020). QuickFacts. < https://www.census.gov/quickfacts/fact/table/CA,US/>.

Chen, J. A., Stevens, C., Wong, S. H. M. \& Liu, C. H. (2019). Psychiatric Symptoms and Diagnoses Among U.S. College Students: A Comparison by Race and Ethnicity. Psychiatric services., 70(6), 442-449. https://doi.org/10.1176/appi.ps.201800388.

Clair JS. (2005) A new model of tracheostomy care: closing the research-practice gap. In: Henriksen, K., Battles, J. B., Marks, E. S., Lewin, D. Advances in patient safety: from research to implementation (Vol. 3: Implementation Issues). Rockville (MD): Agency for Healthcare Research and Quality.

Donato, D. \& Donato, M. (2019). Stages for Undertaking a Systematic Review. Acta Médica Portuguesa. 32(3), 227-235. https://doi.org/10.20344/amp.11923. 
Research, Society and Development, v. 10, n. 9, e47710918369, 2021

(CC BY 4.0) | ISSN 2525-3409 | DOI: http://dx.doi.org/10.33448/rsd-v10i9.18369

Griggs, S. \& Crawford, SL. (2019) Differences in Hope, Core Self-Evaluations, Emotional Well-Being, and Health Risk Behaviors in Freshman University Students. Nursing Forum, 54(4), 505-512.https://doi.org/10.1111/nuf.12364.

Haynes, T. F., Cheney, A. M., Sullivan, J. G., Bryant, K., Curran, J. M., Olson, M., Cottoms, N. \& Reaves, C.(2017) Addressing Mental Health Needs: Perspectives of African Americans Living in the Rural South. Psychiatr Serv. 68(6), 573-578. https://doi.org/10.1176/appi.ps.201600208.

IBGE, Instituto Brasileiro de Geografia e Estatística. (2019) Coordenação de População e Indicadores Sociais. Desigualdades sociais por cor ou raça no Brasil. 41. Disponível em: 〈https://biblioteca.ibge.gov.br/index.php/biblioteca-catalogo?view=detalhes\&id=2101681>. Acesso em: 15 de julho de 2020.

IBGE. Instituto Brasileiro de Geografia e Estatística. (2020) Pesquisa Nacional por Amostra de Domicílios Contínua trimestral, Tabela 6403 - População, por cor ou raça. <https://sidra.ibge.gov.br/tabela/6403>.

Jochman, J. C., Jacob E. Cheadle, J. E., Goosby, B. J., Tomaso, C., Kozikowski, C. \& Nelson, T. (2019) Mental Health Outcomes of Discrimination among College Students on a Predominately White Campus: A Prospective Study. Socius. 5. https://doi.org/10.1177/2378023119842728.

Madeira, Z. \& Gomes, DDO. (2018) Persistentes desigualdades raciais e resistências negras no Brasil contemporâneo. Serv. Soc. Soc. 133, 463-479. https://doi.org/10.1590/0101-6628.154.

McGinley, M. Wolff, J. M., Rospenda, K. M., Liu, L. \& Richman, J. A. (2016). "Risk factors and outcomes of chronic sexual harassment during the transition to college: Examination of a two-part growth mixture model." Social science research. 60: 297-310. https://doi.org/10.1016/j.ssresearch.2016.04.002.

Moher D., Liberati, A., Tetzlaff J. \& Altman D. G. (2009) PRISMA Group. Preferred reporting items for systematic reviews and meta-analyses: the PRISMA statement. PLoS Med.;6(7), e1000097. http://dx.doi.org/10.1371/journal.pmed.1000097.

Oliveira, B. M. C. \& Kubiak, F. (2019) Racismo institucional e a saúde da mulher negra: uma análise da produção científica brasileira. Saúde debate, 43(122), 939-948. https://doi.org/10.1590/0103-1104201912222.

Paveltchuk, F. O. \& Borsa, J. C. (2020). A teoria do estresse de minoria em lésbicas, gays e bissexuais. Rev. SPAGESP, 21(2), 41-54.

Portal Bem Paraná. (2019). No Paraná, apenas 9\% dos alunos de Medicina são negros. https://www.bemparana.com.br/noticia/no-parana-apenas-9-dos-alunosde-medicina-sao-negros\#.X63ar2VKjIV>.

Silva, N. N., Favacho, V. B. C., Boska, G. A., Andrade, E. C., Merces, N. P. \& Oliveira, N. A. F. (2020). Access of the black population to health services: integrative review. Rev. Bras. Enferm., 73(4), e20180834. https://doi.org/10.1590/0034-7167-2018-0834.

Smolen, J. R. \& Araújo, E. M. (2017). Raça/cor da pele e transtornos mentais no Brasil: uma revisão sistemática. Ciênc. saúde coletiva, 22(12), 4021-4030. https://doi.org/10.1590/1413-812320172212.19782016

Sumstine, S., Cruz, S., Schroeder, C., Takeda, S. \& Bavarian, N. (2018) Racial/ethnic variation in mental health correlates of substance use among college students. Journal of Ethnicity in Substance Abuse, 17(2), 94-107. https://doi.org/10.1080/15332640.2017.1300554.

Swift, J. K., Callahan, J. L., Tompkins, K. A., Connor, D. R., \& Dunn, R. (2015) A delay-discounting measure of preference for racial/ethnic matching in psychotherapy. Psychotherapy (Chic). 52(3), 315-20. https://doi.org/10.1037/pst0000019.

Ta Park, V. M. et al. (2020) Promising Results from the Use of a Korean Drama to Address Knowledge, Attitudes, and Behaviors on School Bullying and Mental Health among Asian American College-Aged Students. International journal of environmental research and public health, 17(5), 1637. https://doi.org/10.3390/ijerph17051637

Tikhonov, A. A., Espinosa, A., Huynh, Q.-L., \& Anglin, D. M. (2019). Bicultural Identity Harmony and American Identity Are Associated With Positive Mental Health in U.S. Racial and Ethnic Minority Immigrants. Cultural Diversity and Ethnic Minority Psychology. https://doi.org/10.1037/cdp0000268 